\title{
„Krankheits-Früherkennung ist ein wichtiges Zukunftsthema für die Radiologie“
}

\author{
Interview mit Professor Dr. med. Walter Heindel, Kongresspräsident des 100. Deutschen \\ Röntgenkongresses der Deutschen Röntgengesellschaft und Direktor des Instituts für Klinische \\ Radiologie der Westfälischen Wilhelms-Universität Münster.
}

\section{Was bedeutet es für Sie, Kongresspräsi- dent des 100. Deutschen Röntgenkon- gresses zu sein?}

Das ist für mich auf der einen Seite eine ganz besondere Ehre, aber auf der anderen Seite auch eine Herausforderung: Mein Ziel ist es, dass wir vor allem die nahe Zukunft, die Radiologie im Jahr 2025 , in den Blick nehmen und nicht nur auf die letzten 114 Jahre zurückblicken.

\section{Welche Highlights und fachlichen Schwer- punkte erwarten die Teilnehmer beim 100. Deutschen Röntgenkongress?}

Wir laden alle Teilnehmer gerne ein, bereits am Mittwoch nach Leipzig zu kommen, wenn es um das spannende Zukunftsthema „Radiologie 4.0“ und die Anwendungen neuer Techniken wie z. B. Artificial Intelligence und Machine Learning in der Radiologie gehen wird. Unsere tägliche Arbeit steht vor einem epochalen Wandel: Wir müssen diese neuen Möglichkeiten für uns entdecken und weiterentwickeln, um die Bedeutung unseres Fachs in der Krankenversorgung weiter zu verstärken und zu transformieren. Die Radiologie wird zukünftig zusammen mit anderen Fachgebieten, z. B. der Pathologie, der Genetik, der Labormedizin, und mit neuen methodischen und analytischen Ansätzen entscheidende Weichen für jeden Patienten stellen - Stichwort Integrated Diagnostics. Im Sinne einer personalisierten Medizin - the right patient, the right drug or intervention at the right time - müssen wir unseren klinischen Mehrwert für die moderne Medizin beweisen. In diesem Zusammenhang darf ich besonders auf die Eröffnungsveranstaltung unseres Kongresses mit Ranga Yogeshwar, einem der renommiertesten Wissenschaftsjournalisten in Deutschland, hinweisen. Am Kongress-Freitag steht dann das Thema „Früherkennung“ im Mittelpunkt. Am Samstag besteht für alle Teilnehmer die Möglichkeit, spezielle Fortbildungskurse zu verschiedenen Schwerpunkten der Radiologie wahrzunehmen, um sich weiter zu qualifizieren oder das Wissen aufzufrischen. Durch alle Kongresstage durch zieht sich eine eigens für den Jubiläumskongress konzipierte Highlight-Reihe „Zukunft der Radiologie“. Viele Arbeitsgruppen der Deutschen Röntgengesellschaft werden in speziellen Sitzungen die spannendsten Entwicklungen der nächsten Jahre darstellen. Wer in die Zukunft der verschiedenen Themengebiete oder Tätigkeitsbereiche blicken will, der ist hier richtig. Und nicht zuletzt planen wir für alle Röntgenkongressbesucher eine ganz große Party am Donnerstagabend, sowie für unseren Nachwuchs eine weitere große Feier am Freitagabend.

Sie haben ein Kongressmotto gewählt, das „Einheit in Vielfalt“ lautet. Können Sie das erläutern?

Unser Fach hat sich seit der Entdeckung der Röntgenstrahlen 1895 in mannigfache Richtungen entwickelt. Wir setzten je nach klinischer Fragestellung inzwischen zahlreiche unterschiedliche bildgebende Verfahren zum Teil auch komplementär ein, bis hin zu neuen Ansätzen wie der molekularen Bildgebung. Und trotz oder gerade wegen dieser Divergenz erscheint es mir und uns sehr wichtig, dass wir die Radiologie unter dem wissenschaftlichen Dach der Deutschen Röntgengesellschaft zusammenhalten. Unser gemeinsames Ziel ist immer genauere Diagnostik und minimal-invasive Therapiemöglichkeiten für unsere Patienten sicherzustellen und damit die Radiologie zu einem Nukleus der Patientenversorgung zu machen.

\section{Wenn man sich Ihre Tätigkeitsbereiche} anschaut, decken Sie in der Radiologie ein sehr breites Feld ab. Wieso haben Sie gerade die Früherkennung als Kongressschwerpunkt gewählt?

Ich halte die Früherkennung von Krankheiten für ein weiteres wichtiges Zukunftsthema der Radiologie. Wir leben in einer Gesellschaft, in der wir und vor allem unsere Kinder immer älter werden und es zu

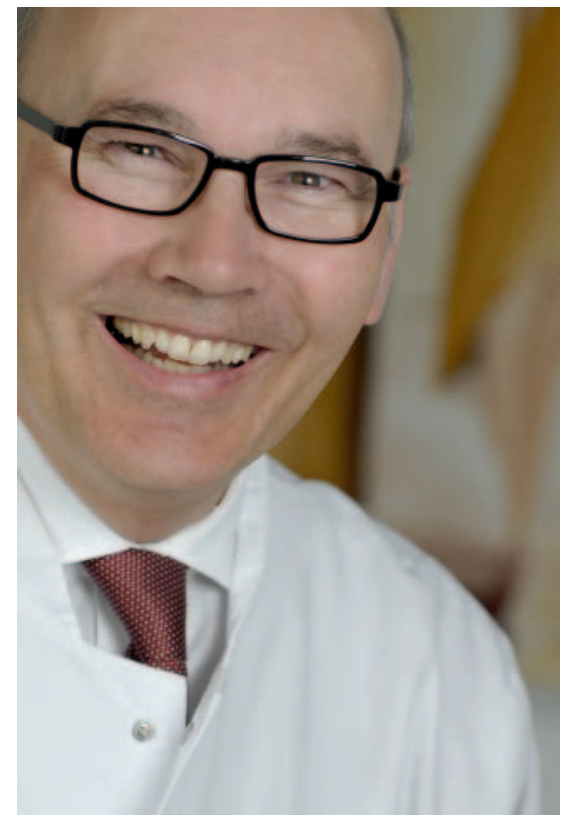

Prof. Dr. med. Walter Heindel

Verschiebungen in den Krankheitsspektren kommt. Mein Ziel ist daher darzustellen und zu diskutieren, welche Systeme der Früherkennung mittels Bildgebung etabliert sind, welche Krankheitsbilder diesbezüglich derzeit evaluiert werden und welche Visionen wir für die Zukunft haben. Status quo sind für mich beispielsweise die intensivierte Früherkennung für Frauen mit genetischen Risiken und das deutsche Mammografie-Screening-Programm. Zu evaluieren ist die Früherkennung beim Lungenkrebs und beim Prostatakarzinom beide Erkrankungen gehören zu den häufigsten Tumorerkrankungen - wie auch die Chancen und Bürden einer Früherkennung beim Morbus Alzheimer. In Zukunft könnten Kombinationen von Labormethoden und Bildgebung wichtig werden. Oder ganz visionär: Kann die Radiologie künftig bereits gefährdete Organe identifizieren, bevor dort eine Metastasierung bei einem Patienten eintritt? 
Sie zählen zu den Pionieren des Mammografie-Screening-Programms (MSP) in Deutschland. Ihr Team der ReferenzScreening-Einheit in Münster gehörte zu den ersten, die mit dem Angebot der Brustkrebs-Früherkennung in der Regelversorgung bereits 2005 starteten. Kann man eventuell inzwischen anhand von Zahlen belegen, dass sich die Brustkrebssterblichkeit reduziert hat?

Zu den Besonderheiten des deutschen Mammografie-Screening-Programms gehören sicherlich die Qualitätssicherung auf allen Ebenen und die konsequente prospektive digitale Dokumentation. Anhand der erfassten Daten kann geprüft werden, ob diese Früherkennungsmaßnahme ihren Zweck erfüllt und wie sie weiterentwickelt werden kann. Alle Parameter, die wir bisher gemessen haben, weisen darauf hin, dass dieses Screening-Programm den Effekt erreichen wird, auf den es hinzielt, nämlich unter anderem die Sterblichkeit an Brustkrebs in Deutschland zu senken. Derzeit sterben pro Jahr immer noch mehr als 17800 Frauen in Deutschland an Brustkrebs. Seit das Screening in Deutschland flächendeckend etabliert wurde, werden mehr Krebsvorstufen und invasive Karzinome in einem frühen Stadium - pT1 - entdeckt. Für Frauen bedeutet dies, dass immer häufiger die betroffene Brust erhalten werden kann und immer seltener eine Chemotherapie notwendig ist. Epidemiologische Analysen belegen inzwischen einen signifikanten Rückgang prognostisch ungünstiger Brustkrebsstadien. Bei wiederholter Screening-Teilnahme beträgt die Abnahme der Inzidenz fortgeschrittener Tumorstadien 20 Prozent. Diese Ergebnisse lassen bereits jetzt darauf schließen, dass durch das Mammografie-Screening-Programm die Sterblichkeitsrate an Brustkrebs in Deutschland gesenkt werden kann.

Wären Sie dann dafür, dass man das Programm eventuell auch auf jüngere und ältere Frauen ausweitet?

Das ist eine weltweit geführte Debatte und auch in Deutschland muss man diskutieren, ob das Screening-Programm für das 50. bis 69. Lebensjahr bis ins 75. Lebensjahr verlängert wird. Unter Berücksichtigung weiterer Ergebnisse sollte auch sehr gründlich evaluiert werden, ob man das Eintrittsalter auf 45 Jahre festlegt. Für darunter liegende Altersstufen macht es nur bedingt Sinn. Brustkrebs ist eigentlich eine Erkrankung des höheren Lebensalters. Beispielsweise wissen wir für Nordrhein-Westfalen, dass bei Frauen unter 50 Jahren nur 18 Prozent aller Mammakarzinome auftreten.

Als ein Nachteil des Mammografie-Screening-Programms wird Überdiagnostik genannt. Wie schätzen Sie das ein?

Überdiagnostik bedeutet, dass eine Tumorart oder Tumorerkrankung durch das Screening diagnostiziert wird, an der ein Mensch nie gestorben wäre. Sowohl im Screening, als auch bei jeder anderen Art von klinischer Diagnostik kann es passieren, dass man etwas diagnostiziert, was vielleicht nicht relevant ist. In der Diskussion geht es darum, wie groß der Anteil an Überdiagnostik ist. Seriöse Studien gehen davon aus, dass dies etwa 10 Prozent der Erkrankungen betrifft. Wir adressieren in einer aktuellen Studie zum Einsatz der Tomosynthese als primäres Screeningverfahren in Kombination mit der sogenannten synthetischen Bildgebung genau diesen Aspekt. Auf der einen Seite wünschen wir uns, so viel wie möglich zu erkennen. Wenn wir mehr erkennen, müssen wir aber auf der anderen Seite auch prüfen, ob dann im Intervall die sogenannten Intervallkarzinome abnehmen. Genau diese schwierige epidemiologische Fragestellung wollen wir in einer aktuellen, von der Deutschen Forschungsgemeinschaft geförderten Studie - ToSyMa - angehen. Weltweit ist diese Frage wissenschaftlich noch nicht beantwortet. Wir glauben, dass wir in Deutschland hier ganz besondere Voraussetzungen haben, um auch diese Frage sehr gezielt beantworten zu können.

Wie bewerten Sie Screenings auf Brustkrebs anhand von Bluttests? Werden diese genetischen Tests über kurz oder lang die radiologischen Verfahren ablösen?

Nein, ich denke, dass es weiterhin bei einer Kombination bleiben wird. Beispielsweise gibt es bereits die sogenannte intensivierte Früherkennung für Frauen, die ein Lebenszeitrisiko bis zu 70 Prozent haben, an Brustkrebs zu erkranken. Hier kommen genetische Tests zum Einsatz. Dabei geht es um die Diagnostik von Brustkrebserkrankungen, die durch bestimmte Genveränderungen hervorgerufen werden. In diesem Zusammenhang sind sicherlich das BRCA1und das BRCA2-Gen sehr bekannt. Darüber hinaus gibt es inzwischen mindestens 8 weitere eindeutig bekannte kritische Gene. Wenn die Genetiker der humangenetischen Beratung ein Vorliegen dieser Gene bestätigen, haben die betroffenen Frauen einen Anspruch, an ausgewählten universitären Zentren eine besondere Früherkennung zu erhalten: Hier kommt dann primär die Magnetresonanztomografie zum Einsatz, ergänzt durch eine Röntgenmammografie und derzeit noch den Ultraschall.

Wenn Sie an die Zukunft der Radiologie denken: Werden sich die bildgebenden Verfahren in die Breite entwickeln - für Screenings - oder Werkzeuge der Präzisionsmedizin sein?

Ich glaube, beides ist wichtig. Neben der Krankheitsfrüherkennung müssen wir zunehmend auch im sogenannten Monitoring von Behandlungen Fortschritte erzielen. Es geht beispielsweise darum, möglichst früh zu erkennen, ob bei Patienten eine eingreifende Behandlung wie eine Chemotherapie den gewünschten Effekt erzielt oder nicht. Wenn man frühzeitig die Therapie anpassen kann, ist das gut für den Patienten und auch das Gesundheitssystem. Denn unter Umständen handelt es sich um sehr teure Therapien. Wir müssen in Zukunft erstens auf eine gezielte und spezifische Bildgebung - Stichwort Targeted Imaging - hinarbeiten. Zweitens glaube ich, dass unser Fach noch nicht am Ende ist. Es gibt neue Techniken, wie beispielsweise die optoakustische Bildgebung. Und drittens werden wir neue Analysemethoden wie künstliche Intelligenz für die Mustererkennung einsetzen. Das alles muss mit anderen diagnostischen Disziplinen wie der Pathologie, der Epidemiologie und den genetischen Techniken kombiniert werden - die Zukunft liegt in einer integrierten Diagnostik!

Das klingt spannend - sollten sich angehende Mediziner deshalb für die Radiologie entscheiden?

Ich glaube, dass wir deswegen ein so spannendes Fach sind, weil wir zunehmend im 
Zentrum der modernen Medizin stehen. Wir müssen über sehr viel Wissen zu den verschiedenen Gebieten und zu den Fragestellungen der Kollegen aus den anderen Disziplinen verfügen. Mit moderner Technik, die man beherrschen muss, können wir oft wesentliche Weichenstellungen für Patienten, sowohl in der Diagnostik, aber auch in der Beratung hinsichtlich der richtigen Therapie, vornehmen. Mir macht es besonders Spaß, an dieser Schlüsselstelle zu arbeiten - ich kann das angehenden Medizinern nur empfehlen. Wen Technik fasziniert und wer sich gleichzeitig auch persönlich um Patienten in oft kritischen Situationen kümmern will, für den ist Radiologie ein tolles Fach.
Wie lautet Ihre persönliche Botschaft für die Besucher des 100. Deutschen Röntgenkongresses?

Schauen Sie mit uns in die Zukunft! Das Fach Radiologie bleibt spannend und es entwickelt sich gerade unglaublich schnell weiter. Auch mit neuen Techniken, die uns als herkömmliche Radiologen noch nicht so bekannt oder vertraut sind. Informieren Sie sich auf diesem besonderen Kongress über künstliche Intelligenz, über neue Früherkennungstechniken und über neue Methoden bei Erkrankungen im Kindesalter, aber auch im hohen Alter.
Der 100. Deutsche Röntgenkongress findet vom 29. Mai bis 1. Juni im Congress-Center Leipzig statt. Weitere Informationen unter https://www. roentgenkongress.de.

Einen Kurzfilm mit dem Kongresspräsidenten Professor Dr. med. Walter Heindel finden Sie online unter https://youtu.be/f_jzhOnO7h0. In diesem geht es um Früherkennung und ihre wachsende Bedeutung in der medizinischen Bildgebung.

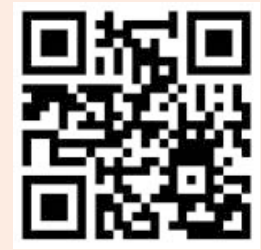

Professor Dr. med. Michael Forsting, ebenfalls Kongresspräsident des 100. Deutschen Röntgenkongresses, spricht im Kurzfilm über das Kongress-Thema „Radiologie 4.0“ und die Bedeutung der Digitalisierung für das Fach. Den Film finden Sie online unter https:// youtu.be/FPQRFCRM5Jg.

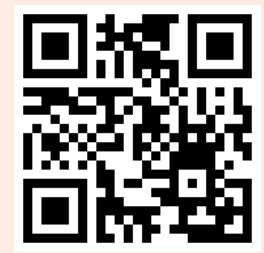

